\title{
Diabetic bullae
}

\author{
RAMAN BHUTANI, SHERNAZ WALTON
}

\begin{abstract}
Bullosis diabeticorum is an uncommon dermatological manifestation of diabetes. Bullae can appear spontaneously in diabetic patients. The majority of patients have pre-existing complications such as nephropathy and neuropathy. The condition is generally self-limiting and the diagnosis is often made clinically with, the appearance of painless, tense blisters arising from non-inflamed skin.

Br J Diabetes Vasc Dis 2015;15:8-10
\end{abstract}

Key words: diabetic bullae, bullosis diabeticorum, diabetes

\section{Introduction}

Diabetic bullae, also known as bullosis diabeticorum, is a spontaneous, distinct, non-inflammatory, blistering condition of the skin predominantly seen in patients with diabetes mellitus with a distal distribution. The condition was first reported in 1930 by Kramer. ${ }^{1}$ Later, Rocca and Pereyra ${ }^{2}$ in 1963 described this lesion as "like burn-induced blister". In 1967, Cantwell and Martz coined the term, "bullosis diabeticorum".

The majority of patients with bullous disease of diabetes have associated nephropathy and neuropathy, leading to the hypothesis of an underlying associated local sub-basement membranezone connective-tissue alteration and micro-angiopathy causing blisters. A lower threshold of suction-induced blister formation ${ }^{4}$ has led to the theory that trauma is a possible aetiological factor. Spontaneous bullae may be the first sign of underlying impaired glycaemic control. ${ }^{5}$

The overall prevalence of diabetic bullae is under-reported. Annual incidence is variable. The incidence in a diabetic population in the United States has been reported to be around $0.5 \%$, being twice as common in males with an age range of 17-84 years. ${ }^{6}$ However, a study from the Indian sub-continent suggested an incidence of $2 \% .^{7}$

\section{Characteristics}

Diabetic bullae are characterised by spontaneous, painless,

Department of Dermatology, Hull and East Yorkshire Hospitals NHS Trust and Hull York Medical School, UK

Address for correspondence: Dr Raman Bhutani Department of Dermatology, Hull and East Yorkshire Hospitals, Anlaby Road, Hull HU3 2JZ, UK

E-mail: raman19dec@gmail.com

http://dx.doi.org/10.15277/bjdvd.2015.004 tense, blisters of variable size measuring $0.5-17 \mathrm{~cm}$ in diameter, containing sterile clear fluid and arising from a non-erythematous base (Figure 1). Onset is often abrupt and can develop overnight, usually without any symptoms; however mild discomfort and a burning sensation have been reported in some patients. ${ }^{7}$ Lesions have a predilection for the distal lower extremities more than the upper extremities, especially the tips of the toes and plantar surfaces of the feet. Truncal involvement is rare but not unknown and is usually associated with involvement of the upper limbs and hands. ${ }^{8,9}$ Spontaneous resolution has been seen in 2-6 weeks without residual pigmentation and scarring unless there is associated secondary infection.

Though less frequent, sub-epidermal variants of bullae can be filled with haemorrhagic fluid and may show scarring and atrophy on resolution due to dermal involvement. ${ }^{8}$

\section{Histopathology}

Histological examination shows bullae with inconsistent levels of skin layer separation. Cleavage can occur at intra- and sub-epidermal levels but combinations of sub-epidermal and supra-basal are not infrequent. ${ }^{8}$ Findings are variable, depending on the age of the blister, due to rapid re-epithelialisation. Interestingly, adjacent epidermis is often unremarkable. ${ }^{10}$ The dermis often shows minimal inflammation and microvascular changes consistent with diabetes. The proteinaceous fluid contained in bullae is often clear and sterile; however the sub-epidermal variant can be haemorrhagic. ${ }^{8}$ Immunopathological features are unremarkable. Non-specific capillary associated immunoglobulin $\mathrm{M}$ and complement $\mathrm{C} 3$ have been reported but no consistent findings have been demonstrated. ${ }^{11}$

\section{Causes}

The pathophysiology of diabetic bullae appears to be multifactorial. There is a lower threshold for suction-induced blister formation in the diabetic population in comparison to controls, and the distal prominence of diabetic bullae has led to speculation for the role of trauma in this group of patients. ${ }^{4}$ However, this fails to account for the absence of these lesions in the vast majority of the diabetic population and the fact that these lesions resolve spontaneously. Some have suggested that the associated micro-angiopathy and sympathetic autonomic denervation leading to impaired microvascular perfusion, predisposes to the premature ageing of connective tissue. ${ }^{12}$ This does not account for the fact that diabetic bullae can also be the first presentation of the pre-diabetic state (impaired glucose tolerance) in some individuals, neither does it explain the absence of bullae in the majority of patients with longstanding and complicated diabetes. ${ }^{5}$ There does not seem to be a consistent 
Figure 1. Tense bulla arising from non-inflamed skin on the foot of a patient with diabetes

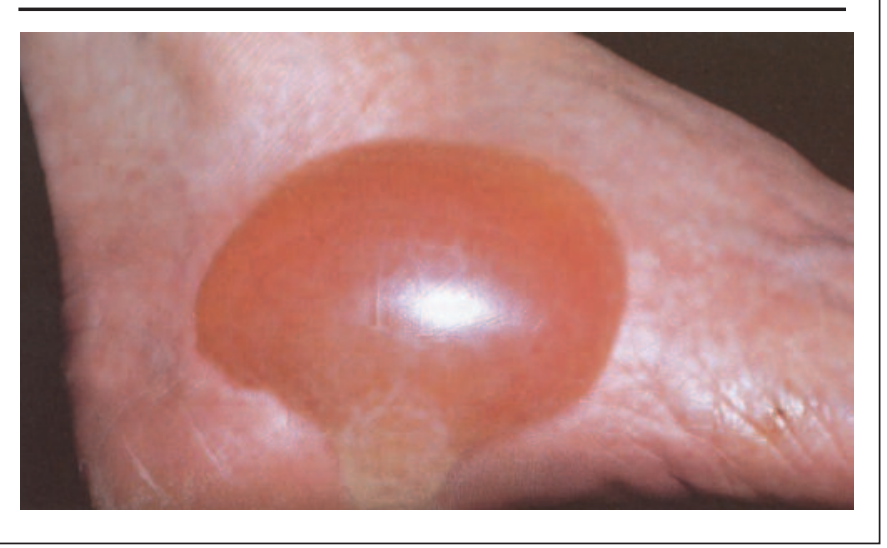

association between the occurrence of bullae and glycaemic control. Disturbances in calcium, magnesium and carbohydrate metabolism have been postulated in the literature,, 8 and excessive exposure to ultraviolet light has also been suggested as a causative factor in a few cases. ${ }^{13}$

\section{Diagnosis and management}

Determining the cause of bullae is challenging and requires meticulous clinical assessment and clinico-pathologic correlation. It is clear that this condition needs to be distinguished from other bullous disorders and therefore a referral to a dermatologist may be required. Exclusion of similar vesiculo-bullous aetiologies must be considered. Thorough clinical history and examination will differentiate between:

- frictional blister,

- oedema blister,

- pemphigoid / pemphigus,

- pseudoporphyrias,

- phytophoto-toxic reaction,

- photo-sensitivities

- and bullous drug eruptions.

Skin biopsy is performed to confirm the histological diagnosis. Fluid culture and sensitivity are undertaken if secondary bacterial infection is suspected, which may warrant appropriate treatment. Absence of primary immune-pathological abnormality and a negative direct immunofluorescence test helps to differentiate this condition from bullous pemphigoid and epidermolysis bullosa acquisita. Protoporphyrin levels help to exclude porphyrias, especially with lesions on the dorsal surface of hands; protoporphyrin levels are often raised in patients with concurrent nephropathy, which may also contribute to the condition.

Ultimately, the condition is self-limiting and usually resolves spontaneously within 2-6 weeks. It requires mainly supportive therapy. Most often, blisters are left intact to avoid introduction of secondary infection. ${ }^{14}$ Fluid aspiration with sterile technique using a large bore needle to prevent accidental de-roofing in very large bullous lesions has been suggested. Aggressive wound care in large de-roofed blisters is required to prevent ulceration. The

\section{Key messages}

- Diabetic bullae are relatively uncommon but are most often associated with suboptimal glycaemic control

- Their presence in a diabetic patient should alert physicians to look for other microvascular problems such as nephropathy and neuropathy

- The aetiology is not completely clear, although trauma may be a predisposing factor in its development

use of antibiotics is warranted if secondary infection is evident. Application of topical emollient with antiseptic can reduce discomfort and prevent infection. Lesions usually heal without residual scarring or post inflammatory pigmentation. Nevertheless, repeated recurrent episodes leading to ulceration ${ }^{15}$ and underlying osteomyelitis, ${ }^{16}$ though rare, have been reported. Bullous symptoms can reoccur over the years - the longest interval of up to a decade has been reported. ${ }^{17}$

\section{Summary}

Recognition of diabetic bullae is important as their differential diagnosis is wide. The risk of subsequent infection is perhaps the most pressing concern in clinical practice. There remains an unclear connection with the degree of glycaemic control, although awareness of other potential vascular complications should always be at the forefront of one's mind and, given that such blisters are generally self-limiting, treatment remains mainly supportive.

\section{Conflict of interest None \\ Funding None.}

\section{References}

1. Kramer DW. Early or warning signs of impending gangrene in diabetes. Med J Rec 1930;132:338-42.

2. Rocca FF, Pereyra E. Phlyctenar lesions in the feet of diabetic patients. Diabetes 1963;12:220-2. http://dx.doi.org/10.2337/diab.12.3.220

3. Cantwell AR Jr, Martz W. Idiopathic bullae in diabetics. Bullosis diabeticorum. Arch Dermatol 1967;96:42-44. http://dx.doi.org/10.1001/archderm.1967.01610010048005

4. Bernstein JE, Levine LE, Medenica MM, Yung CW, Soltani K. Reduced threshold to suction-induced blister formation in insulin-dependent diabetics. J Am Acad Dermatol 1983;8:790-1. http://dx.doi.org/10.1016/S0190-9622(83)80007-3

5. Lopez PR, Leicht S, Justin R, Sigmon JR, Stigall L. Bullosis diabeticorum associated with prediabetic state. South Med J 2009;102:643-4. http://dx.doi.org/10.1097/SMJ.0b013e3181a506d6

6. Poh-Fitzpatrick MB, Elston DM, Junkins-Hopkins JM. Bullous disease of diabetes. Medscape 20 December 2013. http://emedicine.medscape. com/article/1062235-overview (accessed 19th June 2014).

7. Mahajan S, Koranne RV, Sharma SK. Cutaneous manifestation of diabetes mellitus. Indian J Dermatol Venereol Leprol 2003;69:105-08.

8. Toonstra J, Utercht MD. Bullosis diabeticorum: Report of a case with a review of literature. J Am Acad Dermatol 1995;13:799-805 http://dx.doi.org/10.1016/ S0190-9622(85)70226-5

9. Renoux M, Guy-Brand B, Tutin M, Bour H. Dermatose bulleuse apparemment idiopatique chez un diabetique. Soc Fr Dermatol 1969;76:165-8.

10. Kerl H, Kresbach: Zu einigen Aspekten der "Bullosis Diabeticorum". Hautarzt 1974;25:60-5. 
11. James WD, Odom RB, Goette DK. Bullous eruption of diabetes mellitus. A case with positive immunofluorescence microscopy findings. Arch Dermatol 1980;116:1191-2. http://dx.doi.org/10.1001/archderm.1980. 01640340101029

12. Bravemann IM, Keh-Yen A. Ultrastructural abnormalities of the microvasculature and elastic fibres in the skin of juvenile diabetics. J Invest Dermatol 1984;82: 270-4. http://dx.doi.org/10.1111/1523-1747. ep12260279

13. Bernstein JE, Medenica M, Soltani K, Griem SF. Bullous eruption of diabetes mellitus. Arch Dermatol 1979;115:324-5. http://dx.doi.org/ 10.1001/archderm.1979.04010030032012
14. Lipsky BA, Baker PD, Ahroni JH. Diabetic bullae: 12 cases of a purportedly rare cutaneous disorder. Int J Dermatol 2000;39:196-200. http://dx.doi.org/10.1046/j.1365-4362.2000.00947.x

15. Larsen K, Jensen T, Karlsmark T, Holstein PE. Incidence of bullosis diabeticorum - a controversial cause of chronic foot ulceration. Int Wound J 2008;5:591-6. http://dx.doi.org/10.1111/j.1742-481X.2008.00476.x

16. Tunuguntla A, Patel KN, Peiris AN, Zakaria WN. Bullosis diabeticorum associated with osteomyelitis. Tenn Med 2004;97(11):503-4.

17. Dobozy A, Husz S, Schneider I, Szabó. Bullous dermatosis associated with latent diabetes: A case report. Dermatologica 1972;144:283-6. http://dx.doi.org/10.1159/000252138

\title{
$\triangle A B C D$ RECRUITING NOW iabesIty
}

\section{Do you have patients with type 2 diabetes and obesity needing better control, despite previous liraglutide use?}

REVISE-Diabesity is a randomised controlled trial which offers the real chance of improved glycaemic control and reduced weight to enrolled participants, who will be randomised to: 1) Liraglutide 1.8mg, 2) Endobarrier, 3) Endobarrier + liraglutide

Endobarrier is an endosopically inserted duodenal-jejunal bypass liner which early studies suggest might lead to considerable weight loss and improved glycaemic control

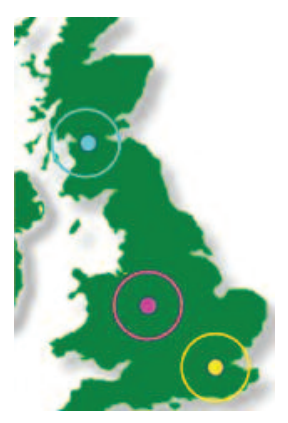

\author{
To refer patients (Glasgow/ Birmingham / London) \\ please contact: \\ Dr Piya Sen Gupta, \\ ABCD Research Fellow \\ Email: revise.diabesity@nhs.net \\ Mobile: 07866319487
}

Please see the study website (includes selection criteria):

http://www.diabetologists-abcd.org.uk/research/endobarrier_study.htm

ISRCTN00151053, NCT02055014 